\title{
THE
}

$5-1-1989$

\section{Quantum and Nonlocal Coherent Effects in Boltzmann Gases}

Alexander E. Meyerovich

University of Rhode Island, sfo101@uri.edu

Follow this and additional works at: https://digitalcommons.uri.edu/phys_facpubs

Terms of Use

All rights reserved under copyright.

\section{Citation/Publisher Attribution}

Meyerovich, A. E.. (1989). Quantum and Nonlocal Coherent Effects in Boltzmann Gases, Phys. Rev. B, 39(13), 9318-9329. doi: 10.1103/PhysRevB.39.9318

Available at: http://dx.doi.org/10.1103/PhysRevB.39.9318

This Article is brought to you for free and open access by the Physics at DigitalCommons@URI. It has been accepted for inclusion in Physics Faculty Publications by an authorized administrator of DigitalCommons@URI. For more information, please contact digitalcommons-group@uri.edu. 


\title{
Quantum and nonlocal coherent effects in Boltzmann gases
}

\author{
A. E. Meyerovich \\ Northwestern University, Department of Physics and Astronomy, 2145 Sheridan Road, Evanston, Illinois 60208
}

(Received 11 October 1988)

\begin{abstract}
Quantum coherent phenomena in Boltzmann gases are studied on the basis of the generalized Waldmann-Snider kinetic equation. The equation takes into account the effects of quantum identity of particles. The conditions are formulated for the propagation of different collective modes in gases of particles with arbitrary internal degrees of freedom. Collective coherent effects are macroscopic manifestations of the molecular field for the transverse (off-diagonal) elements of particle distribution in a "polarized" system (a system with a nonuniform population of internal energy levels in an unperturbed state). The simplest results are obtained for particles with equidistant internalenergy levels exploiting the analogy with spin-polarized quantum gases. The accurate study of nonlocal interaction terms permits one to compare classical-kinetic and Fermi-liquid approaches to kinetic phenomena in dilute gases, and shows the limitations of both approaches. In the case of collective modes, more accurate schemes of derivation of the kinetic equation must be accompanied by the simultaneous calculations of nonlocal contributions.
\end{abstract}

\section{INTRODUCTION}

Quantum peculiarities in the properties of gases with a classic Boltzmann distribution function may be caused by different effects. The simplest ones are due to the complicated structure of the internal energy spectrum of particles (e.g., large molecules) and to the necessity of quantum calculations of two-particle scattering amplitudes and cross sections. In many cases such as transport phenomena the rest of the calculations are performed classically. Moreover, it is often assumed that, between the collisions, particles remain in pure quantum internal states, and all the kinetic phenomena in principle may be described using the classical Boltzmann kinetic equation for some multicomponent system where the roles of different components are played by particles in different pure internal states (the so-called Wang Chang and Uhlenbeck approach ${ }^{1}$ ). This set of equations may be solved on the basis of well-developed techniques like Chapman-Enskog or Grad expansions. Such an approach gives perfect results for dilute Boltzmann gases if the interaction is diagonal in internal states, and we are interested in the processes (like thermal conductivity or viscosity) averaged over internal states of particles.

More intriguing are the quantum effects associated not with quantum peculiarities of cross sections, but with symmetry properties of wave functions, quantum identity of particles, and noncommutativity of different singleparticle or two-particle operators. Such effects may be exhibited if the operator of two-particle interaction and/or the nonequilibrium single-particle density matrix are nondiagonal in single-particle internal states (the equilibrium density matrix is diagonalized simultaneously with the Hamiltonian). Such off-diagonal components of the single-particle density matrix describe oscillations of the particle between the corresponding internal states. Macroscopically these single-particle oscillations sometimes may be exposed in the form of small oscillation contributions to the basically diffusive propagation of some macroscopic variables or even in the form of some specific collective modes, providing that the corresponding internal energy levels are degenerate or nearly degenerate so that the transitions between them are not accompanied by a very large energy emission and absorption.

Apart from that, the macroscopic manifestations of single-particle oscillations demand the sufficient coherence of the interaction. The molecular field is caused by the coherent part of the interaction which must be observable on the background of the mainly noncoherent dephasing interaction responsible for the dissipation and suppression of the macroscopic consequences of singleparticle oscillations. In the usual pictures of dilute Boltzmann gases the particles are absolutely independent between the collisions, and the collisions are supposed to be completely dephasing ones, so that none of the coherent effects may survive. It is not always true.

It is well known that the molecular field is mainly linear in the interaction (linear in the scattering amplitude), while the collision integral responsible for the dissipation (being proportional to the scattering probabilities) is quadratic in scattering amplitude. Therefore, the coherent manifestations of the molecular field may be noticeable when the interaction effects may be described by some sort of expansion in the (small) scattering amplitude). One example of mean-field coherent oscillations is represented by plasma oscillations when the plasma is considered as collisionless because of the rapid decrease of the Coulomb scattering amplitude with the increase of particle velocities (temperature).

The other example is represented by the so-called "quantum gases"- gases of particles with the thermal de Broglie wavelengths which are comparable with or even larger than the scattering amplitude (atomic dimension). As the most striking manifestation of strong coherent collective phenomena in such gases one may mention the propagation of spin waves in spin-polarized low- 
temperature hydrogen or helium gases with Boltzmann distribution functions.

However, the observation of macroscopic coherent phenomena in Boltzmann gases vitally depends not only on the relative value of the molecular field, but also on the type and spectrum of single-particle operators. For example, if the internal states of particles are classified by nuclear-spin variables exclusively, the corresponding phenomena have already been studied rather thoroughly (see, e.g., reviews ${ }^{2}$ and references therein).

One of the main aims of this paper is to specify the necessary conditions on the single-particle variables which would allow one to expect appearance of some macroscopic collective quantum effects in Boltzmann gases.

Usually the quantum effects in the kinetics of gases of particles with an arbitrary spectrum of internal states are described on the basis of Waldmann-Snider kinetic equation (see review ${ }^{1}$ and references therein). However, the standard Waldmann-Snider kinetic equation cannot be used for our purposes - it does not take into account the quantum identity of particles, and thus omits all manifestations of different "exchange" effects, which are vitally important (as we will see below) for the existence of coherent collective modes. This shortcoming of the standard form of the Waldmann-Snider kinetic equation is to some extent responsible for the considerable suspicion encountered by the theoretical predictions of the possibility of spin-wave propagation in spin-polarized Boltzmann gases.

Below, the quantum identity of particles will be taken into account explicitly. This clarifies the general class of quantum collective phenomena represented in the particular case of spin-polarized gases by the spin-wave propagation.

There are two general approaches to the description of dilute Boltzmann gases. The first one is a purely classical kinetic approach on the basis of some sort of Boltzmann kinetic equation. The second one is more heuristic and is based on a theory of the Fermi-liquid type. The latter approach to dilute Boltzmann gases is quite reasonable, since in these gases all the interaction effects are quadra- tic in distribution functions $\hat{f}(\mathbf{p})$ and may be described by the term $\hat{F}\left(\mathbf{p}, \mathbf{p}^{\prime}\right) \hat{f}(\mathbf{p}) \hat{f}\left(\mathbf{p}^{\prime}\right)$. The only remaining operation is to relate the interaction function $\hat{F}\left(\mathbf{p}, \mathbf{p}^{\prime}\right)$ (the analog of the Fermi-liquid function) to the two-particle scattering amplitude. Such a concept of "statistical quasiparticles" gives excellent results (see, e.g., Ref. 3) reproducing, for example, thermodynamic virial corrections sometimes more easily than the classical kinetic approach. In kinetics both approaches also give very similar results. This may be illustrated by the example of spin-wave propagation in quantum gases studied by means of both Fermi-liquid-like theory ${ }^{2,4-6}$ and the classical kinetic approach of Ref. 7.

However, the more detailed analysis reveals some minor but still disturbing discrepancies in the forms of the kinetic equations assumed by these two approaches. The discrepancies occur mainly in the zero-sound-like terms which quite naturally appear in the Landau type of kinetic equation, but are absent in the Boltzmann one. The study of nonlocal interaction effects undertaken below gives one the clear understanding of the interrelations between the different approaches and indicates the precise limitations for both of them. Besides, the study of the nonlocal gradient terms is absolutely necessary since we are dealing mainly with the $k^{2}$ spectrum of collective modes.

The paper has the following structure. In Sec. II we reexamine the Waldmann-Snider kinetic equation for identical particles. The conditions of appearance of macroscopic coherent quantum oscillations are studied in Sec. III for Boltzmann gases of particles with arbitrary internal structure. The nonlocal gradient corrections and the corresponding limitations of different approaches are dealt with in Sec. IV. The main results are briefly discussed in the Summary (Sec. V).

\section{KINETIC EQUATION}

As a rule, all kinetic phenomena in dilute Boltzmann gases of particles with arbitrary internal degrees of freedom are discussed using the so-called Waldmann-Snider kinetic equation $^{1}$

$$
\begin{aligned}
\partial_{t} \hat{f}+(i / \hbar)[\hat{H}, \hat{f}]=(2 \pi)^{4} \hbar^{2} \int d \mathbf{p}_{1}\left\{\int d \mathbf { p } ^ { \prime } \left[\hat{T}^{\dagger}\left(\mathbf{g}^{\prime}, \mathbf{g}\right) \hat{f}\left(\mathbf{p}^{\prime}\right) \hat{f}\left(\mathbf{p}_{1}^{\prime}\right) \delta(E) \hat{T}\left(\mathbf{g}, \mathbf{g}^{\prime}\right)\right.\right. \\
\left.\left.-\widehat{f}(\mathbf{p}) \widehat{f}\left(\mathbf{p}_{1}\right) \hat{T}^{\dagger}\left(\mathbf{g}, \mathbf{g}^{\prime}\right) \delta(E) \hat{T}\left(\mathbf{g}^{\prime}, \mathbf{g}\right)\right]-(i / 2 \pi)\left[\hat{T}(\mathbf{g}, \mathbf{g}), \hat{f}(\mathbf{p}) \hat{f}\left(\mathbf{p}_{1}\right)\right]\right\},
\end{aligned}
$$

where $\widehat{f}$ is the Wigner distribution function $(\widehat{f}$ is still a matrix in the space of internal states, $\widehat{T}$ is the scattering $T$ matrix, $\mathbf{g}=\mathbf{p}_{1}-\mathbf{p},[\cdots, \cdots]$ is the commutator of the corresponding operators, $\hat{H}$ is the single-particle Hamiltonian, and $\delta(E)$ reflects the energy conservation for collisions. Above, in contrast to the standard form of the Waldmann-Snider kinetic equation, we had already used the optical theorem

$$
\widehat{T}^{\dagger}-\widehat{T}=2 \pi i \hat{T}^{\dagger} \delta\left(E-H_{12}^{(0)}\right) \widehat{T},
$$

where $\hat{H}_{12}^{(0)}$ is the Hamiltonian for two noninteracting particles.

We are interested in the collective quantum effects 
which are caused by the molecular field. The molecular field contains all terms linear in $\hat{T}$ (and some of the quadratic terms) in the kinetic equation. The linear terms may possess any sign in contrast to dissipative terms (which are always quadratic in $T$ and have the fixed sign). Such linear terms lead to some dissipationless oscillation effects masked by dissipative-diffusion motion, and, in some exceptional cases, even to the possibility of propagation of low-damped collective waves. It is evident, that the considerable molecular field (the last term in figure brackets) may be expected according to Eq. (2.1) only if the $T$ matrix does not commute with the single-particle Wigner distribution functions. Therefore the molecular field in Eq. (2.1) vanishes if the interaction does not depend on the single-particle internal states, and the $T$ matrix has a form

$$
T_{\beta v}^{\alpha \mu}=t \delta_{\beta}^{\alpha} \delta_{v}^{\mu}
$$

Here each of the indices denotes the whole set of indices characterizing the internal states of particles. Such an interaction corresponds to the conservation law for populations of internal states, and is a purely "exchange" one in the sense of internal states. For real molecules the approximation (2.3) is rather good when one assumes as internal all atomic and molecular states except for some electronic terms responsible for the interaction.

The absence of the molecular field in the standard Waldmann-Snider kinetic Eq. (2.1) in the case of the "exchange" interaction (2.3) obviously contradicts, e.g., the well-known data ${ }^{2,4-7}$ on kinetics of spin-polarized gases for which the molecular field is extremely important and leads to the existence of the spin waves in the case of an exchange (in the sense of nuclear-spin states) interaction.

The above example clearly demonstrates that Eq. (2.1) does not take into account all quantum effects and must be modified. The main shortcoming of the usual derivations of the Waldmann-Snider kinetic equation (2.1) is caused by not taking into account the effects of quantum identity of particles (some results for identical particles had already been obtained in Ref. 8).

Below we briefly outline the scheme of derivation of the kinetic equation for identical particles, and study the results of quantum identity of particles. In principle, the analogous kinetic equation had been derived by Lhuillier and Laloë, ${ }^{7}$ but only for the local exchange interaction of particles with nuclear spins (without any other internal degrees of freedom. Some qualitative remarks on coherent modes in gases of particles with internal degrees of freedom has been given in Ref. 9.). Our equation will be free of these limitations.

There exist many ways to derive the kinetic equation with different degrees of accuracy, and an incredibly large amount of papers describing different subtle effects caused by, e.g., various decoupling procedures for correlation functions or by slightly different concepts of molecular chaos. That is why for the sake of clarity we will deliberately restrict ourselves and assume the simplest and the roughest scheme of derivation of kinetic equation. It will cost us lack of accuracy, especially in Sec. IV, but most of the results will have a very transparent form. Afterwards it will be possible to get more accurate results applying some of the well-known and complicated derivation procedures.

The first two equations of the Bogoliubov-BornGreen-Kirkwood-Yvon (BBGKY) hierarchy for one- and two-particle density matrices have the form

$$
\begin{aligned}
& i \hbar \partial_{t} \hat{\rho}_{1}=\hat{L}_{1} \hat{\rho}_{1}+\operatorname{Tr}_{2} \hat{L}_{12}^{(i)} \hat{\rho}_{12}, \\
& i \hbar \partial_{t} \hat{\rho}_{12}=\hat{L}_{12} \hat{\rho}_{12}+\operatorname{Tr}_{3} \hat{L}_{123}^{(i)} \hat{\rho}_{123},
\end{aligned}
$$

where $\hat{L}$ are the Liouville operators, and the interaction is taken into account only in $\hat{L}^{(i)}$ operators. If instead of a more accurate decoupling procedure one simply neglects the three-particle term, then

$$
\begin{aligned}
\rho_{12}(t)= & \exp \left[-i H_{12}\left(t-t_{0}\right) / \hbar\right] \rho_{12}\left(t_{0}\right) \\
& \times \exp \left[i H_{12}\left(t-t_{0}\right) / \hbar\right]
\end{aligned}
$$

where $H_{12}$ is the two-particle Hamiltonian.

At this point it is always assumed that before the collision $\left(t_{0} \rightarrow-\infty\right)$ there are no correlations, and the twoparticle density matrix may be factorized

$$
\rho_{12}\left(t_{0}\right)=\rho_{1}\left(t_{0}\right) \rho_{2}\left(t_{0}\right) \text {. }
$$

However, Eq. (2.6) holds only for nonidentical particles. Since the two-particle wave function for identical noninteracting particles has the form

$$
\Psi_{12}^{\alpha \beta}=\left(\frac{1}{2}\right)^{1 / 2}\left(\psi_{1}^{\alpha} \psi_{2}^{\beta} \pm \psi_{2}^{\alpha} \psi_{1}^{\beta}\right),
$$

the two-particle density matrix must be factorized as

$$
\rho_{\beta v}^{\alpha \mu}=\rho_{\beta}^{\alpha} \rho_{v}^{\mu} \pm \rho_{v}^{\alpha} \rho_{\mu}^{\beta}
$$

instead of (2.6). Here the indices $\alpha, \beta, \mu$, and $v$ characterize both the internal states and spatial coordinates or momenta of particles. In other respects the derivation practically does not deviate from the standard ones. One has to go to the limit $t_{0} \rightarrow-\infty$, to use the molecular chaos hypothesis and to introduce the $T$ matrix by the equation

$$
\widehat{T}=\hat{V}+\hat{V} \hat{G} \hat{T},
$$

where $\hat{V}$ is the two-particle interaction operator, and the free two-particle propagator $\hat{G}=\left(E-\hat{H}_{12}^{(0)}+i 0\right)^{-1}$ is determined by the Hamiltonian of noninteracting particles $\hat{H}_{12}^{(0)}=\hat{H}_{12}-\hat{V}$. As a result, the kinetic equation for a single-particle density matrix $\rho_{\beta}^{\alpha}$ takes the form

$$
\partial_{t} \rho_{\beta}^{\alpha}+(i / \hbar)[H, \rho]_{\beta}^{\alpha}=(i / \hbar)\left(-T_{\gamma \nu}^{\alpha \mu} \rho_{\beta \mu}^{\gamma v}+\rho_{\gamma v}^{\alpha \mu} T_{\beta \mu}^{+\gamma v}-T_{\gamma v}^{\alpha \mu} \rho_{\lambda k}^{\gamma v} T_{\eta \delta}^{+\lambda k} G_{\beta \mu}^{+\eta \delta}+G_{\gamma \nu}^{\alpha \mu} T_{\lambda k}^{\gamma v} \rho_{\eta \delta}^{\lambda k} T^{+\eta}{ }_{\beta \mu}^{\delta}\right),
$$

where $\hat{H}$ is the single-particle Hamiltonian (without the interaction), and the density matrix $\rho_{\beta \nu}^{\alpha \mu}$ must be factorized according to Eq. (2.7). If one uses the factorization (2.6) instead of (2.7), then Eq. (2.9) reduces to the usual WaldmannSnider kinetic equation (2.1).

Equations (2.9) and (2.7) are rather cumbersome. Since this paper's only interest is the mean-field effects and not the 
dissipative terms, we will follow only terms linear in $\hat{T}$ which contribute to the mean field exclusively (though the molecular field certainly also contains the contributions quadratic in $\hat{T}$ which become very important in the case of strong interaction).

Equations (2.9) and (2.7) give the kinetic equation for the gas of identical particles with internal degrees of freedom. At first let us separate the spatial and internal degrees of freedom. Assuming the momentum conservation in the infinite homogeneous space we get

$$
\widehat{T}\left(\mathbf{p}, \mathbf{p}^{\prime} ; \mathbf{p}_{1}, \mathbf{p}_{1}^{\prime}\right)=\widehat{T}\left(\mathbf{g}^{\prime}, \mathbf{g}\right) \delta\left(\mathbf{p}+\mathbf{p}_{1}-\mathbf{p}^{\prime}-\mathbf{p}_{1}^{\prime}\right),
$$

where $\mathbf{g}=\mathbf{p}_{1}-\mathbf{p}$ is the momentum of relative motion, $\mathbf{g}^{\prime}=\mathbf{p}_{1}^{\prime}-\mathbf{p}^{\prime}$. It is convenient to consider Eq. (2.9) in the momentum representation. Then this equation may be written as

$$
\begin{aligned}
\partial_{t} \rho_{\beta}^{\alpha}\left(\mathbf{p}, \mathbf{p}^{\prime \prime}\right)+(i / \hbar)[\hat{H}, \hat{\rho}]_{\beta}^{\alpha}\left(\mathbf{p}, \mathbf{p}^{\prime \prime}\right) \\
=(i / \hbar) \int d \mathbf{p}_{1} d \mathbf{p}^{\prime}\left\{-T_{\gamma \nu}^{\alpha \mu}\left(\mathbf{p}+\mathbf{p}_{1}-2 \mathbf{p}^{\prime}, \mathbf{p}_{1}-\mathbf{p}\right)\left[\rho_{\beta}^{\gamma}\left(\mathbf{p}^{\prime}, \mathbf{p}^{\prime \prime}\right) \rho_{\mu}^{\nu}\left(\mathbf{p}+\mathbf{p}_{1}-\mathbf{p}^{\prime}, \mathbf{p}_{1}\right) \pm \rho_{\mu}^{\gamma}\left(\mathbf{p}^{\prime}, \mathbf{p}_{1}\right) \rho_{\beta}^{\nu}\left(\mathbf{p}+\mathbf{p}_{1}-\mathbf{p}^{\prime}, \mathbf{p}^{\prime \prime}\right)\right]\right. \\
\left.+\left[\rho_{\gamma}^{\alpha}\left(\mathbf{p}, \mathbf{p}^{\prime}\right) \rho_{\nu}^{\mu}\left(\mathbf{p}_{1}, \mathbf{p}_{1}-\mathbf{p}^{\prime}+\mathbf{p}^{\prime \prime}\right) \pm \rho_{\nu}^{\alpha}\left(\mathbf{p}, \mathbf{p}_{1}-\mathbf{p}^{\prime}+\mathbf{p}^{\prime \prime}\right) \rho_{\gamma}^{\mu}\left(\mathbf{p}_{1}, \mathbf{p}^{\prime}\right) T^{+\gamma \nu}\left(\mathbf{p}_{1}-\mathbf{p}^{\prime \prime}, \mathbf{p}_{1}-2 \mathbf{p}^{\prime}+\mathbf{p}^{\prime \prime}\right)\right]\right\}+\cdots,
\end{aligned}
$$

where the indices denote now only the internal variables, and $\cdots$ stands for the terms quadratic in $\widehat{T}$ (it is worth repeating that the lack of explicit expressions of quadratic terms do not imply that these terms are necessarily small and may be neglected).

In the next step one has to introduce Wigner distribution functions $\hat{f}(\mathbf{r}, \mathbf{p})$ which are still matrices in the space of internal variables:

$$
\begin{aligned}
& f_{\beta}^{\alpha}(\mathbf{r}, \mathbf{p})=(2 \pi \hbar)^{-3} \int d \mathbf{q} \exp (-i \mathbf{q r} / \hbar) \rho_{\beta}^{\alpha}(\mathbf{p}, \mathbf{p}-\mathbf{q}), \\
& \rho_{\beta}^{\alpha}(\mathbf{p}, \mathbf{p}-\mathbf{q})=\int d \mathbf{r} \exp (i \mathbf{q r} / \hbar) f_{\beta}^{\alpha}(\mathbf{r}, \mathbf{p}) .
\end{aligned}
$$

As a result, Eq. (2.10) reduces to

$$
\begin{aligned}
\partial_{t} \hat{f}+(i / \hbar)[\hat{H}, \hat{f}]= & i i / \hbar)(2 \pi \hbar)^{-3} \\
\times & \int d \mathbf{p}_{1} d \mathbf{q}^{\prime} d \mathbf{q}^{\prime \prime} d \mathbf{r}^{\prime} d \mathbf{r}^{\prime \prime} \exp \left[(i / \hbar)\left(\mathbf{q}^{\prime} \mathbf{r}^{\prime}+\mathbf{q}^{\prime \prime} \mathbf{r}^{\prime \prime}\right)\right] \\
& \times\left[-\hat{T}\left(\mathbf{g}+\mathbf{q}^{\prime \prime}, \mathbf{g}-\mathbf{q}^{\prime \prime}\right) \hat{f}\left(\mathbf{r}+\mathbf{r}^{\prime}, \mathbf{p}-\mathbf{q}^{\prime \prime}\right) \hat{f}\left(\mathbf{r}+\mathbf{r}^{\prime \prime}, \mathbf{p}_{1}\right) \mp \hat{T}\left(-\mathbf{g}-\mathbf{q}^{\prime}, \mathbf{g}-\mathbf{q}^{\prime}\right) \hat{f}\left(\mathbf{r}+\mathbf{r}^{\prime}, \mathbf{p}_{1}\right) \widehat{f}\left(\mathbf{r}+\mathbf{r}^{\prime \prime}, \mathbf{p}-\mathbf{q}^{\prime}\right)\right. \\
& +\hat{f}\left(\mathbf{r}+\mathbf{r}^{\prime}, \mathbf{p}\right) \hat{f}\left(\mathbf{r}+\mathbf{r}^{\prime \prime}, \mathbf{p}_{1}\right) \hat{T}+\left(\mathbf{g}+\mathbf{q}^{\prime}+\mathbf{q}^{\prime \prime}, \mathbf{g}+\mathbf{q}^{\prime}-\mathbf{q}^{\prime \prime}\right) \\
& \left. \pm f\left(\mathbf{r}+\mathbf{r}^{\prime}, \mathbf{p}\right) f\left(\mathbf{r}+\mathbf{r}^{\prime \prime}, \mathbf{p}_{1}\right) \hat{T}+\left(\mathbf{g}+\mathbf{q}^{\prime}+\mathbf{q}^{\prime \prime},-\mathbf{g}-\mathbf{q}^{\prime}+\mathbf{q}^{\prime \prime}\right)\right]+\cdots,
\end{aligned}
$$

where the indices for $\hat{f}$ and $\hat{T}$ operators must be written exactly in the same way as in Eq. (2.10).

Usually one is interested only in slightly inhomogeneous situations such as transport problems or a propagation of long-wave oscillations. Then it is worth introducing the gradient expansion in the right-hand side of Eq. (2.12) using the relation

$$
\begin{aligned}
\int d \mathbf{r}^{\prime} d \mathbf{q} \exp \left(i \mathbf{q} \mathbf{r}^{\prime} / \hbar\right) f\left(\mathbf{r}+\mathbf{r}^{\prime}, \mathbf{q}\right) \phi(\mathbf{q})=(2 \pi \hbar)^{3}\{ & f(\mathbf{r}, \mathbf{q}) \phi(\mathbf{q})+i \hbar\left(\partial / \partial q_{i}\right)\left[\phi(\mathbf{q})\left(\partial / \partial r_{i}\right) f(\mathbf{r}, \mathbf{q})\right] \\
& \left.-\left(\hbar^{2} / 2\right)\left(\partial^{2} / \partial q_{i} \partial q_{k}\right)\left[\phi(\mathbf{q})\left(\partial^{2} / \partial r_{i} \partial r_{k}\right) f(\mathbf{r}, \mathbf{q})\right]+\cdots\right\}_{\mathbf{q} \rightarrow 0} .
\end{aligned}
$$

In ordinary transport calculations it is enough to use only the first term in the expression (2.13). Such a kinetic equation does not take into account any nonlocal effects in the interaction and assumes the form

$$
\begin{aligned}
\partial_{t} \hat{f}+(i / \hbar)[\hat{H}, \hat{f}]_{\beta}^{\alpha}=(i / \hbar)(2 \pi \hbar)^{3} \int d \mathbf{p}_{1}\{ & -T_{\gamma \nu}^{\alpha \mu}(\mathbf{g}, \mathbf{g}) f_{\beta}^{\gamma}(\mathbf{r}, \mathbf{p}) f_{\mu}^{\nu}\left(\mathbf{r}, \mathbf{p}_{1}\right) \mp T_{\gamma \nu}^{\alpha \mu}(-\mathbf{g}, \mathbf{g}) f_{\mu}^{\gamma}\left(\mathbf{r}, \mathbf{p}_{1}\right) f_{\beta}^{\nu}(\mathbf{r}, \mathbf{p}) \\
& \left.+f_{\gamma}^{\alpha}(\mathbf{r}, \mathbf{p}) f_{v}^{\mu}\left(\mathbf{r}, \mathbf{p}_{1}\right)\left[T_{\beta \mu}^{+\gamma \nu}(\mathbf{g}, \mathbf{g}) \pm T_{\beta \mu}^{+v \gamma}(\mathbf{g},-\mathbf{g})\right]\right\}+\cdots
\end{aligned}
$$

Equation (2.14) must replace the Waldmann-Snider kinetic equation (2.1) in the case of identical particles.

If the interaction does not depend on internal states of colliding particles, then the $T$ operator corresponds to an "exchange" interaction (2.3), and Eq. (2.14) may be simplified:

$$
\begin{aligned}
& \partial_{t} f_{\beta}^{\alpha}+(i / \hbar)[\hat{H}, \hat{f}]_{\beta}^{\alpha}=(i / \hbar)(2 \pi \hbar)^{3} \int d \mathbf{p}_{1}\left\{2 f_{\beta}^{\alpha}(\mathbf{p}) f_{\mu}^{\mu}\left(\mathbf{p}_{1}\right) t_{0} \pm 2 f_{\mu}^{\alpha}(\mathbf{p}) f_{\beta}^{\mu}\left(\mathbf{p}_{1}\right) t_{1} \pm\left[\hat{f}(\mathbf{p}), \hat{f}\left(\mathbf{p}_{1}\right)\right]_{\beta}^{\alpha} t(-\mathbf{g}, \mathbf{g})\right\}+\cdots, \\
& 2 t_{0}=t^{\dagger}(\mathbf{g}, \mathbf{g})-t(\mathbf{g}, \mathbf{g}), \quad 2 t_{1}=t^{\dagger}(\mathbf{g},-\mathbf{g})-t(-\mathbf{g}, \mathbf{g}) .
\end{aligned}
$$

Not all the terms in Eq. (2.15) are really linear in $\hat{T}$; according to the optical theorem (2.2) all the terms on the rhs of Eq. (2.15) except the one with the commutator are in reality quadratic in $T$. Therefore, the terms linear in $T$ in the col- 
lision operator in kinetic equation (2.15) may always be rearranged as

$$
\begin{aligned}
& \partial_{t} \hat{f}_{\beta}^{\alpha}+(i / \hbar)[\hat{H}, \hat{f}]_{\beta}^{\alpha}=(i / \hbar)(2 \pi \hbar)^{3} \int d \mathbf{p}_{1}\left[f(\mathbf{p}), f\left(\mathbf{p}_{1}\right)\right]_{\beta}^{\alpha} t_{2}+\cdots, \\
& 2 t_{2}= \pm t(-\mathbf{g}, \mathbf{g}) \pm t^{+}(\mathbf{g},-\mathbf{g}) .
\end{aligned}
$$

When indices $\alpha, \beta, \ldots$ correspond only to the nuclearspin states, the kinetic equation (2.16) is equal to the Lhuillier-Laloë kinetic equation. ${ }^{7}$ Equation (2.12)-(2.14) generalize the results of Ref. 7 to the cases of nonlocal and/or nonexchange interaction and to the cases of particles with arbitrary internal degrees of freedom.

\section{QUANTUM OSCILLATIONS AND DIFFUSION OF PARTICLE DISTRIBUTIONS OVER INTERNAL STATES: COLLECTIVE MODES}

In this section we will study only the systems with local interaction (2.14). Since there are no zero-sound terms in the kinetic equation for the isotropic gas systems with local interaction (2.14), all inhomogeneities and perturbations of internal-state populations propagate in the diffusive manner with a quadratic spectrum $\omega \propto k^{2}$. If one adopts a classic or semiclassic approach (such as the Wang Chang and Uhlenbeck approach ${ }^{1}$ ) for the particles in pure (diagonal) states, then such processes are described by macroscopic equations

$$
\partial_{t} N_{\alpha}=-D_{\alpha \beta}^{i k} \partial^{2} N_{\beta} / \partial r_{i} \partial r_{k}
$$

in the corresponding macroscopic densities, while the matrix of diffusion coefficients $D_{\alpha \beta}^{i k}$ is positive definite. Certainly, only quadratic combinations $T^{+} T$ with a fixed sign may contribute to $\hat{D}$. The linear in $\hat{T}$ terms (which have no fixed sign) are neglected within semiclassical approaches. These terms are responsible for some oscillations accompanying diffusive motion, and make contributions to the imaginary parts of the diffusion coefficients. Below we are only interested in such contributions.

Note, that the collective oscillation effects appear only for macroscopic quantities which correspond to nondiagonal (in internal indices) components of the density matrix. Generally speaking, the kinetic equation decouples, in the case of an "exchange" interaction (2.3), into two separate subsystems for diagonal and off-diagonal components of the single-particle distribution. The interaction terms in the kinetic equation (2.12) in $f_{\beta}^{\alpha}$ contain, in the case of an "exchange" interaction, the distribution functions only in the form

$$
\widehat{A} f_{\beta}^{\alpha} f_{\mu}^{\mu}+\widehat{B} f_{\mu}^{\alpha} f_{\beta}^{\mu},
$$

where the integral operators $\hat{A}$ and $\widehat{B}$ do not depend on internal variables and are free of corresponding indices. Being linearized in small deviations $f_{\beta}^{(1) \alpha}$ from the equilibrium $f_{\beta}^{(0) \alpha}$ the expression (3.2) reduces to

$$
\widehat{A}\left(f_{\beta}^{(1) \alpha} f_{\mu}^{(0) \mu}+f_{\beta}^{(0) \alpha} f_{\mu}^{(1) \mu}\right)+\widehat{B}\left(f_{\mu}^{(1) \alpha} f_{\beta}^{(0) \mu}+f_{\mu}^{(0) \alpha} f_{\beta}^{(1) \mu}\right) \text {. }
$$

It is natural to assume that the particles in equilibrium occupy pure internal states and that the equilibrium distribution $f_{\beta}^{(0) \alpha}$ is diagonal in internal indices $f_{\beta}^{(0) \alpha}=f^{(0)} \delta_{\beta}^{\alpha}$. As a result, Eq. (3.3) takes the form

$$
\left(\hat{A} \delta_{\mu}^{\mu}+2 \widehat{B}\right) f^{(0)} f_{\beta}^{(1) \alpha}+\widehat{A} f^{(0)} \delta_{\beta}^{\alpha} f_{\mu}^{(1) \mu},
$$

meaning that the equation in diagonal components of the distribution $f_{\beta}^{(1) \alpha}(\alpha=\beta)$ contains only diagonal components of $\hat{f}^{(1)}$, and the equation in $f_{\beta}^{(1) \alpha}(\alpha \neq \beta)$ contains only the off-diagonal components of $\hat{f}^{(1)}$.

Therefore, the subsystems of kinetic equations for diagonal and nondiagonal components of distribution $\hat{f}^{(1)}$ decouple from each other completely. Following the usual notations for spin-polarized systems, it is possible to call the equation for diagonal components of $\hat{f}^{(1)}$ the longitudinal kinetic equation and call the equation for nondiagonal components - the transverse kinetic equation (in the case of spin-polarized systems the terms longitudinal and transverse mean the dynamics of longitudinal and transverse components of magnetization, respectively).

The complete decoupling of longitudinal and transverse equations makes it possible for "exchange" systems to obtain the very accurate longitudinal kinetic results on the basis of the semiclassical Wang Chang and Uhlenbeck approach ${ }^{1}$ briefly described above (providing the accurate symmetrization procedures for the collision operator are applied). Certainly, all molecular field effects [linear in the $\widehat{T}$ terms of Eq. (2.12)] disappear from the longitudinal kinetic equation for exchange systems (2.3) with local interaction (2.15); all the commutators in internal variables vanish (in this case all the operators are diagonal in internal indices), and the only nonvanishing commutators in (2.12) are associated with spatial derivatives, i.e., with nonlocal (gradient) interaction terms. As a result, the longitudinal kinetic equation in the macroscopic limit reduces to the ordinary diffusion equation (3.1).

Some of the important oscillation effects [the commutator in Eq. (2.16)] disappear from the standard Waldmann-Snider kinetic equation (2.1) for nonidentical particles. It is easily illustrated for internal-spin variables: Eq. (2.1) describes only the diffusive and dissipative behavior of spin perturbations ${ }^{10}$ (spin relaxation), while taking into account the quantum identity of particles [the nonzero commutator in the right-hand side of Eq. (2.16)] leads in many cases even to the possibility of propagation of low-damped spin waves. ${ }^{2,4-7}$

Let us analyze the simplest examples of quantum oscillation effects on Boltzmann gases with an "exchange" interaction (2.3) independent of internal single-particle states. Since the interaction parameters depend mainly on the electron terms and not on, say, vibrational, rotational, or spin nuclear states, such an "exchange" approximation (2.3) seems quite reasonable.

Generally we are interested in damped oscillations with the quadratic spectrum $\omega \propto k^{2}$, where $\omega$ is the frequency and $k$ is the wave vector of the oscillations. Therefore, one has to apply the nonlocal kinetic equation (2.12) with 
the gradient terms up to the second order in expansion (2.13). However, in this section only the local equation (2.16) will be used, mainly for the sake of clarity. The nonlocal contributions will be analyzed in the next section.

If there are $Z$ single-particle internal states (including degenerate ones), all single-particle operators are $Z \times Z$ matrices. In many cases the effective number $Z$ may be decreased considerably using symmetry arguments, discussing the perturbations of different symmetries separately, exploiting the possible large differences in energy or frequency gaps for different modes, and averaging over insignificant internal variables.

It is convenient to introduce some matrix basis in the space of internal variables, i.e., in the space of matrices $Z \times Z$. One may choose as basis $Z$ diagonal matrices $\hat{l}_{0 s}=\hat{L}_{z}^{s}(s=0,1, \ldots, Z-1)$ and $Z^{2}-Z$ nondiagonal matrices $\hat{l}_{t s}=\hat{L}_{+}^{t} L_{z}^{s} \quad(t=1,2, \ldots, Z-1 ; s=0,1, \ldots, Z$ $-t-1)$, where the operators $\hat{L}_{z}, \hat{L}_{+}=\hat{L}_{x}+i \hat{L}_{y}$ are the matrices of the components of the angular momentum operator $\hat{L}, L=(Z-1) / 2$.

All single-particle operators $\hat{A}$ may be expanded over the basis set $\hat{l}_{i}$,

$$
\widehat{A}=A_{\beta}^{\alpha}=A_{i} l_{i \beta}^{\alpha}=A_{i} \hat{l}_{i},
$$

while the products and the commutators of basis operators can be represented as

$$
\begin{aligned}
& \hat{l}_{i} \hat{l}_{j}=a_{i j k} \hat{l}_{k}, \quad\left[\hat{l}_{i}, \hat{l}_{j}\right]=b_{i j k} \hat{l}_{k}, \quad b_{i j k}=a_{i j k}-a_{j i k}=-b_{j i k} \\
& \operatorname{Tr} \hat{l}_{i}=l_{i \alpha}^{\alpha}=c_{i}
\end{aligned}
$$

using the well-known commutation relations for the angular momentum operators.

Certainly, the equilibrium (undisturbed) Wigner distribution function $f_{\beta}^{(0) \alpha}$ and single-particle Hamiltonian may be considered as diagonal in internal states

$$
\hat{H}=\left(p^{2} / 2 M\right) \hat{l}_{00}+h_{s} \hat{l}_{0 s}, \quad \hat{f}^{(0)}=(2 \pi \hbar)^{-3} n_{s}^{(0)}(\mathbf{p}) \hat{l}_{0 s}
$$

where the functions $n_{s}^{(0)}$ are the trivial linear combinations of an equilibrium (Boltzmann) distribution function of particles in pure internal states $\bar{n}_{\lambda}$. The disturbed distribution function $\hat{f}$ can contain all $Z^{2}$ operators $\hat{l}_{i}$ :

$$
\widehat{f}=\hat{f}^{(0)}+v_{i} \hat{l}_{i}, \quad v_{i}=-n_{i}^{(0)} \phi_{i} / T
$$

where $T$ is the temperature. [For diagonal matrix elements the definition of $n_{i}^{(0)}$ in (3.7) is the same as in (3.6); for the off-diagonal elements the corresponding $n^{(0)}$ is to be defined as $\left(\bar{n}_{\lambda} \bar{n}_{\mu}\right)^{1 / 2}$.]

Usually the single-particle internal spectrum consists of different groups of internal states clearly separated either by large gaps or symmetry conservation laws. If one is interested in perturbations with the symmetry inherent to one of these groups then it is possible to eliminate all other internal states by taking the corresponding traces, thus decreasing the effective dimensionality $Z$.

With the help of Eq. (3.5)-(3.7) the linearized kinetic equation $(2.16)$ reduces to

$$
\left[(\omega-\mathbf{k v}) I_{k i}-\Omega_{k i}^{(0)}+\Omega_{k i}^{(i)}\right] v_{i} \widehat{l}_{k}+\beta_{k i} \hat{l}_{k} \int d \mathbf{p}_{1} v_{i}\left(\mathbf{p}_{1}\right) t_{2}=-i \Gamma_{k}\left(v_{i}\right) \widehat{l}_{k},
$$

with the notations

$$
\begin{aligned}
& \hat{\Gamma}=\Gamma_{k} \hat{l}_{k}, \\
& \hat{I}=I_{i k}=\delta_{i k}, \quad \hat{\Omega}^{(0)}=\Omega_{k i}^{(0)}=(i / \hbar) b_{j i k} h_{j}, \\
& \hat{\Omega}^{(i)}=\Omega_{k i}^{(i)}(\mathbf{p})=(1 / \hbar) b_{i j k} \int d \mathbf{p}_{1} n_{j}^{(0)}\left(\mathbf{p}_{1}\right) t_{2}, \\
& \hat{\beta}_{k}=\beta_{k i}(\mathbf{p})=(i / \hbar) b_{j i k} n_{j}^{(0)}(\mathbf{p})
\end{aligned}
$$

( $\mathbf{v}=\mathbf{p} / M$ is the velocity of particles), while the operator $\widehat{\Gamma}$, being composed by all terms linearized in $v$ and quadratic in $\widehat{T}$ in the kinetic equation, is a matrix in the indices $(i, k)$ and an integral operator in momenta space. The operator $\hat{\Gamma}$ contains some of the contributions to the molecular field (quadratic in $\widehat{T}$ ), and all of the dissipative diffusion and relaxation terms.

The above frequencies (3.9) have the following physical meaning. The frequencies $\Omega_{i k}^{(0)}$ are determined by the distances $h_{i}$ between the single-particle internal levels (without the interaction), and characterize the gaps in the single-particle oscillation spectra for the off-diagonal components of the distribution. The frequencies $\Omega^{(i)}$ determine the oscillation frequencies for the off-diagonal components of the distribution in the (linear in $\widehat{T}$ ) internal molecular field given by the coherent part of the interaction.

Multiplying Eq. (3.8) by all basis matrices $\hat{l}_{i}$ and calculating the corresponding traces one arrives at

$\left[(\omega-\mathbf{k v}) \hat{I}-\hat{\Omega}^{(0)}+\hat{\Omega}^{(i)}\right] \boldsymbol{v}+\hat{\beta} \int d \mathbf{p}_{1} t_{2} \boldsymbol{v}\left(\mathbf{p}_{1}\right)=-i \hat{\Gamma}(\boldsymbol{v})$,

where the vector $v$ has the components $v_{i}$ (3.7). The exact solution of Eq. (3.10) is nearly always impossible due to the complicated structure of the integral operators involved.

Let us determine the long-wave oscillation spectrum and the macroscopic equations of motions corresponding to Eq. (3.10). Since the operator structure of $T$ in Eq. (2.3) reflects some conservation law for the internal states (an exchange" interaction in the sense of internal states), the integrations of Eq. (3.10) over momenta eliminates all the terms related to the interaction

$$
\begin{aligned}
& \left(\omega \hat{I}-\hat{\Omega}^{(0)}\right) \mathbf{N}-k_{i} \mathbf{J}_{i}=0, \\
& \mathbf{N}=(2 \pi \hbar)^{-3} \int d \mathbf{p} \boldsymbol{v} ; \quad \mathbf{J}_{i}=(2 \pi \hbar) \int d \mathbf{p} v_{i} \boldsymbol{v} .
\end{aligned}
$$


In principle, Eq. (3.11) is quite obvious from the beginning: It is the simplest macroscopic conservation law for the off-diagonal densities $\mathbf{N}$ [for the internal-states conserving interaction (2.3)] relating their changes to the corresponding currents $\mathbf{J}_{i}$. However, it is important that the interaction terms in the right-hand and left-hand sides of Eq. (3.10) vanish separately throughout the momenta integration since for $t_{2}(\mathbf{g})=t_{2}(-\mathbf{g})$

$$
\begin{aligned}
\int d \mathbf{p} & \hat{\Omega}^{(i)} \boldsymbol{v}+\int d \mathbf{p} \hat{\beta} \int d \mathbf{p}_{1} t_{2} \boldsymbol{v}\left(\mathbf{p}_{1}\right) \\
= & \left(b_{i j k}+b_{k i j}\right) \int d \mathbf{p} d \mathbf{p}_{1} t_{2} n_{j}^{(0)}(\mathbf{p}) v_{k}\left(\mathbf{p}_{1}\right)=0 .
\end{aligned}
$$

According to Eq. (3.11) the eigen spectrum $\omega_{s}^{(0)}$ at $k=0$ is determined solely by the gaps in the internal spectrum of single-particle noninteracting Hamiltonian

$$
\operatorname{Det}\left\|\omega_{s}^{(0)} \hat{I}-\widehat{\Omega}^{(0)}\right\|=0 \text {. }
$$

In order to evaluate the spectrum at $k \neq 0$ it is necessary to derive another macroscopic equation relating the currents $\mathbf{J}$ to the gradients of densities $\boldsymbol{v}$. It seems hardly possible in a general form; to simplify the problem one may assume the following natural parametrization of the operator $\hat{\Gamma}$ :

$$
\int d \mathbf{p} \widehat{\Gamma}(\boldsymbol{v})=0, \quad(2 \pi \hbar)^{-3} \int d \mathbf{p} v_{i} \widehat{\Gamma}(\boldsymbol{v})=-\hat{\tau}^{-1} \mathbf{J}_{i},
$$

thus introducing the matrix of inverse relaxation times $\hat{\tau}^{-1}$. Since the parametrization (3.14) reflects only the relaxation contributions to $\hat{\Gamma}$, the quadratic-in- $\hat{T}$ contributions to the molecular field either have to be added to the "internal" frequencies $\Omega^{(i)}$ Eq. (3.9) or neglected.

The second macroscopic equation appears after multiplication of Eq. (3.10) by the velocity $v_{i}$ and integration over momenta. Unfortunately, this equation together with Eq. (3.11) form a closed system of equations in only a few cases. One such case corresponds to the so-called "quantum" gases ${ }^{2}$ when the $\hat{T}$ matrix elements do not depend on the particles momenta due to the large wavelengths of particles. More often, one has to use a variational approach (see, e.g., Ref. 7), neglecting higher-order angular harmonics of the functions $\phi$ (3.7):

$$
\phi=\phi^{(0)}+\mathbf{p}_{i} \phi_{i}^{(1)},
$$

where the components $\phi_{i}^{(0),(1)}$ of the vectors $\phi^{(0),(1)}$ are supposed to be constants. Then the second macroscopic equation takes the form

$\left(\omega \hat{I}-\widehat{\Omega}^{(0)}+\widehat{\widehat{\Omega}}^{(i)}-i \hat{\tau}^{-1}\right) \mathbf{J}_{\alpha}-(T / M) k_{\alpha} \mathbf{N}=0$,

and the averaged internal frequency $\widehat{\bar{\Omega}}^{(i)}$ was introduced as

$$
\begin{aligned}
\bar{\Omega}_{i k}=\frac{b_{i j k}}{\hbar M T} \int & d \mathbf{p} d \mathbf{p}_{1} \frac{1}{(2 \pi \hbar)^{3}} n_{j}^{(0)}\left(\mathbf{p}_{1}\right) n_{k}^{(0)}(\mathbf{p}) \\
\times\left[\frac{p^{2}}{3} t_{2}^{(0)}\right. & \\
& \left.\quad-\frac{p p_{1}}{9} t_{2}^{(1)}\right] / \int d \mathbf{p} \frac{1}{(2 \pi \hbar)^{3}} n_{k}^{(0)}(\mathbf{p})
\end{aligned}
$$

(there is no summation over $k$ ), while the angular harmonics of the function $t_{2}(\mathrm{~g})$ are equal to

$t_{2}(\mathbf{g})=\sum_{n} t_{2}^{(n)}\left(p, p_{1}\right) P_{n}(\cos \chi), \quad \cos \chi=\mathbf{p p}_{1} / p p_{1}$,

$P_{n}$ being the Legendre polynomials. Besides, in deriving (3.16) we have exploited the fact that the mean velocity of particles of a Boltzmann gas is determined solely by the temperature and does not depend on their internal states.

The dispersion relation is obtained from Eqs. (3.11) and (3.16):

$$
\operatorname{Det}\left\|\left(\omega \hat{I}-\widehat{\Omega}^{(0)}+\hat{\bar{\Omega}}^{(i)}-i \hat{\tau}^{-1}\right)\left(\omega \hat{I}-\widehat{\Omega}^{(0)}\right)-k^{2}(T / M) \hat{I}\right\|=0 .
$$

Since at $k=0$ the eigen frequencies $\omega_{s}^{(0)}$ are given by Eq. (3.13), the small corrections $\omega_{s}^{(1)}$ to $\omega_{s}^{(0)}$ are, according to Eq. (3.19), quadratic in $k$ in the long-wave limit: $\omega_{s}^{(1)}=k^{2}(T / M) \lambda_{s}^{-1}$, where $\lambda_{s}$ is determined by the equations

$$
\operatorname{Det}\left\|\left[\hat{I}+\left(\omega_{s}^{(0)} \hat{I}-\hat{\Omega}^{(0)}+\widehat{\bar{\Omega}}^{(i)}-i \hat{\tau}^{-1}\right)^{-1}\left(\omega_{s}^{(0)} \hat{I}-\widehat{\Omega}^{(0)}-\lambda_{s} \hat{I}\right)\right] k^{2}(T / M)+\lambda_{s}\left(\omega_{s}^{(0)} \hat{I}-\widehat{\Omega}^{(0)}\right)\right\|=0
$$

(there is no summation over $s$ ). The order of magnitude of parameters $\lambda_{s}$ is $\left|\lambda_{s}\right| \sim\left|\widetilde{\Omega}^{(i)}-i / \tau\right|$.

The perturbations are long-wave or "hydrodynamic" ones if

$\left|\omega^{(1)} /\left(\Omega^{(i)}-i / \tau\right)\right|, k(T / M)^{1 / 2} /\left|\bar{\Omega}^{(i)}-i / \tau\right| \ll 1$.

When the molecular field is strong enough $\bar{\Omega}^{(i)} \tau \gg 1$, the problem is a hydrodynamic one practically for all values of $\omega \tau$. Note, that for strong molecular fields $\Omega^{(i)} \tau \gg 1$, the dissipation beyond the long-wave limit (3.21) is dominated by Landau damping related to the imaginary parts of integrals of the following type (for details see, e.g., Ref. 2):

$$
\begin{aligned}
& \int \frac{L(\mathbf{p}) d \mathbf{p}}{\omega-\mathbf{k v}+\Omega^{(i)}-i 0} \\
& \quad=f \frac{L(\mathbf{p}) d \mathbf{p}}{\omega-\mathbf{k v}+\Omega^{(i)}}-i \pi \int L(\mathbf{p}) \delta\left(\omega-\mathbf{k v}+\Omega^{(i)}\right) d \mathbf{p} .
\end{aligned}
$$

The macroscopic equation of motion associated with the spectrum (3.19) and (3.20) can be reduced to the form

$$
\begin{aligned}
& \left(i \widehat{I} \partial_{t}-\widehat{\Omega}^{*}\right) N=0, \\
& \Omega_{i k}^{*}=\delta_{i k}\left[\omega_{k}^{(0)}-(T / M) \lambda_{k}^{-1} \frac{\partial^{2}}{\partial \mathbf{r}^{2}}\right],
\end{aligned}
$$

using the appropriate choice of the basis in the space of 
matrices involved [there is no summation over index $k$ in Eq. (3.22)].

The dynamics of macroscopic "densities" $\mathbf{N}$ is a combination of three types of coupled processes: the "fast" oscillations with frequencies $\Omega^{(0)}$ in the "external" field $h$, Eq. (3.6) and (3.9), and coupled oscillation-diffusion propagation of spatial perturbations with diffusion matrix $\hat{D}=(T / M) \hat{\tau}$ and "internal" frequencies $\widehat{\Omega}{ }^{(i)}$. Since the internal molecular field is $\Omega^{(i)} \propto t_{2}$ and the relaxation times are $1 / \tau \propto t^{2}$, the condition of the propagation of low-damped collective modes $\Omega^{(i)} \tau \gg 1$ is reduced to the inequality

$$
t M^{3 / 2} T^{1 / 2}<<1
$$

while in the opposite limit $\bar{\Omega}^{(i)} \tau<<1$, the strong collision damping prevents the propagation of collective modes, and the perturbations propagate in the diffusive regime (3.1).

The problem becomes quite simple when we are interested in perturbations with some specific symmetry (3.7) so that the corresponding equation of motion decouples from the rest of equations (3.10), and the macroscopic equations (3.11) and (3.16) are not matrix but scalar ones. Such is the case of internal-spin variables when one is interested in the perturbations of the magnetic moment $\mathbf{M}$ only in the form $M_{x}+i M_{y}$. In such cases one deals only with scalar frequencies $\boldsymbol{\Omega}^{(0)}$ and $\bar{\Omega}^{(i)}$ and relaxation times $\tau$. Then the solutions of one-dimensional Eqs. (3.13) and (3.20) have the trivial form

$$
\omega^{(0)}=\Omega^{(0)}, \lambda=\bar{\Omega}^{(i)}-i / \tau,
$$

and the macroscopic equation of motion (3.21) obtains the Leggett's form ${ }^{11}$

$$
\begin{aligned}
& {\left[i \partial_{t}-\Omega_{0}^{(0)}+(D / \tau)\left(\bar{\Omega}^{(i)}-i / \tau\right)^{-1}\left(\partial^{2} / \partial \mathrm{r}^{2}\right)\right] N=0,} \\
& D=(T / M) \tau .
\end{aligned}
$$

Such a simplification always take place when one is dealing with a gas of particles with equidistant energy levels. Then the single-particle Hamiltonian and equilibrium (unperturbed) density matrix (3.6) have the form

$$
\begin{aligned}
& \hat{H}=\left(p^{2} / 2 M\right) \hat{l}_{00}+h_{0} \hat{l}_{01}, \\
& \hat{f}^{(0)}=(2 \pi \hbar)^{-3} n_{0}(\mathbf{p})\left[\hat{l}_{00}+\left(h_{0} / T\right) \hat{l}_{01}\right] .
\end{aligned}
$$

In this case the equation of motion for the perturbations of the symmetry $\delta \hat{f}=w(\mathrm{p})\left(\hat{L}_{x}+i \hat{L}_{y}\right)$ decouples completely from the rest of the system (3.10), and the results obtain the simplest form (3.23) and (3.24). Such perturbations propagate in the same manner as spin waves in spin-polarized gases. ${ }^{2}$ Note, that this simplification always takes place in the case of a gas of two-level particles.

\section{NONLOCAL AND GRADIENT CONTRIBUTIONS: FERMI-LIQUID ANALOGY}

The gradient expansion (2.13) for the kinetic equation (2.12) makes it possible to take into account the nonlocality of the interaction. In practice such an expansion is equivalent to the expansion of the integrand in the righthand side of Eq. (2.12) in small q. Since we are interested in the corrections to the spectrum Eq. (3.20) $\omega^{(1)} \propto k^{2}$ and to the diffusionlike equations (3.1) and (3.22), it seems necessary to provide this expansion up to the secondorder gradients, i.e., up to the terms of the order $\mathbf{q}^{2}$.

Without gradient corrections, the system of Eqs. (3.11) and (3.16) we are interested in has the form

$\left[\left(\omega_{s}^{(1)}+\omega_{s}^{(0)}\right) \hat{\boldsymbol{I}}-\hat{\boldsymbol{\Omega}}^{(0)}\right] \mathbf{N}-k_{\alpha} \mathbf{J}_{\alpha}=0$,

$\left[\left(\omega_{s}^{(1)}+\omega_{s}^{(0)}\right) \hat{I}-\widehat{\Omega}^{(0)}+\hat{\mathbf{\Omega}}^{(i)}-i \hat{\tau}^{-1}\right] \mathbf{J}_{\alpha}-(T / M) k_{\alpha} \mathbf{N}=0$.

Symmetry considerations within the variational approach (3.15), given by

$$
\begin{aligned}
& v_{i}=\left(n_{i}^{(0)} / N_{i}^{(0)}\right)\left(N_{i}+\mathbf{J}_{i} \mathrm{p} / T\right), \\
& N_{i}^{(0)}=\int n_{i}^{(0)} d \mathbf{p} /(2 \pi \hbar)^{3},
\end{aligned}
$$

[current and density are given by Eqs. (3.11)], demonstrate that the gradient and nonlocal corrections may lead, in the frame of the "exchange" interaction (2.3), only to the following changes of Eqs. (4.1):

$$
\begin{aligned}
& {\left[\left(\omega_{s}^{(1)}+\omega_{s}^{(0)}\right) \hat{I}-\hat{\Omega}^{(0)}\right] \mathbf{N}-\left(\hat{I}+\hat{A}_{1}\right) k_{\alpha} \mathbf{J}_{\alpha}-k^{2} \hat{A}_{3}(T / M) \mathbf{N}=0,} \\
& {\left[\left(\omega_{s}^{(0)}+\omega_{s}^{(1)}\right) \hat{I}-\hat{\Omega}^{(0)}+\hat{\Omega}^{(i)}-i \hat{\tau}^{-1}\right] \mathbf{J}_{\alpha}-(T / M)\left(\hat{I}+\widehat{A}_{2}\right) k_{\alpha} \mathbf{N}=0,}
\end{aligned}
$$

while the corrections different from matrices $\hat{A}_{1,2,3}$ result only in the higher-order (in $k$ ) terms in the collective-mode spectrum.

Now the spectrum $\omega_{s}^{(1)}=k^{2}(T / M) \lambda_{s}^{-1}$ takes the rather cumbersome form

$$
\begin{aligned}
\operatorname{Det} \| \lambda_{s}\left(\omega_{s}^{(0)} \hat{I}-\hat{\Omega}^{(0)}\right)+k^{2}(T / M)\{\hat{I}+ & \left(\hat{I}+\hat{A}_{1}\right)\left(\omega_{s}^{(0)} \hat{I}-\hat{\Omega}^{(0)}+\hat{\Omega}^{(i)}-i \hat{\tau}^{-1}\right)^{-1} \\
& \left.\times\left[\left(I+A_{1}\right)^{-1}\left(\omega_{s}^{(0)} \hat{I}-\widehat{\Omega}^{(0)}\right)-\lambda_{s}\left(\hat{I}+\widehat{A}_{2}\right)\right]-\lambda_{s} \hat{A}_{3}\right\} \|=0 .
\end{aligned}
$$

In order to simplify over complicated general formulas one may restrict oneself to the "exchange" approximation (2.3). Then the expression in the right-hand side of Eq. (2.12) may be rewritten as 


$$
\begin{aligned}
-t & \left(\mathbf{g}+\mathbf{q}^{\prime \prime}, \mathbf{g}-\mathbf{q}^{\prime \prime}\right)\left[f_{\beta}^{\alpha}\left(\mathbf{r}+\mathbf{r}^{\prime}, \mathbf{p}-\mathbf{q}^{\prime \prime}\right)-f_{\beta}^{\alpha}\left(\mathbf{r}+\mathbf{r}^{\prime}, \mathbf{p}\right)\right] f_{\mu}^{\mu}\left(\mathbf{r}+\mathbf{r}^{\prime \prime}, \mathbf{p}_{1}\right) \\
& \mp t\left(-\mathbf{g}-\mathbf{q}^{\prime}, \mathbf{g}-\mathbf{q}^{\prime}\right) f_{\mu}^{\alpha}\left(\mathbf{r}+\mathbf{r}^{\prime}, \mathbf{p}_{1}\right)\left[f_{\beta}^{\mu}\left(\mathbf{r}+\mathbf{r}^{\prime \prime}, \mathbf{p}-\mathbf{q}^{\prime}\right)-f_{\beta}^{\mu}\left(\mathbf{r}+\mathbf{r}^{\prime \prime}, \mathbf{p}\right)\right] \\
& +f_{\beta}^{\alpha}\left(\mathbf{r}+\mathbf{r}^{\prime}, \mathbf{p}\right) f_{\mu}^{\mu}\left(\mathbf{r}+\mathbf{r}^{\prime \prime}, \mathbf{p}_{1}\right)\left[t^{+}\left(\mathbf{g}+\mathbf{q}^{\prime}+\mathbf{q}^{\prime \prime}, \mathbf{g}+\mathbf{q}^{\prime}-\mathbf{q}^{\prime \prime}\right)-t\left(\mathbf{g}+\mathbf{q}^{\prime \prime}, \mathbf{g}-\mathbf{q}^{\prime \prime}\right)\right] \\
& \mp t\left(-\mathbf{g}-\mathbf{q}^{\prime}, \mathbf{g}-\mathbf{q}^{\prime}\right) f_{\mu}^{\alpha}\left(\mathbf{r}+\mathbf{r}^{\prime}, \mathbf{p}_{1}\right) f_{\beta}^{\mu}\left(\mathbf{r}+\mathbf{r}^{\prime \prime}, \mathbf{p}\right) \\
& \pm t^{+}\left(\mathbf{g}+\mathbf{q}^{\prime \prime}+\mathbf{q}^{\prime},-\mathbf{g}+\mathbf{q}^{\prime \prime}-\mathbf{q}^{\prime}\right) f_{\mu}^{\alpha}\left(\mathbf{r}+\mathbf{r}^{\prime}, \mathbf{p}\right) f_{\beta}^{\mu}\left(\mathbf{r}+\mathbf{r}^{\prime \prime}, \mathbf{p}_{1}\right) .
\end{aligned}
$$

As a result, the terms linear in gradients in the right-hand side of Eq. (2.12) take the following form in accordance with Eq. (2.13):

$$
\begin{aligned}
-(2 \pi \hbar)^{3} \int d \mathbf{p}_{1}[ & t(\mathbf{g}, \mathbf{g}) \frac{\partial}{\partial \mathbf{p}} f_{\beta}^{\alpha}(\mathbf{r}, \mathbf{p}) \nabla f_{\mu}^{\mu}\left(\mathbf{r}, \mathbf{p}_{1}\right) \pm t(-\mathbf{g}, \mathbf{g}) \nabla f_{\mu}^{\alpha}\left(\mathbf{r}, \mathbf{p}_{1}\right) \frac{\partial}{\partial \mathbf{p}} f_{\beta}^{\mu}(\mathbf{r}, \mathbf{p}) \\
& +f_{\beta}^{\alpha}(\mathbf{r}, \mathbf{p}) \nabla f_{\mu}^{\mu}(\mathbf{r}, \mathbf{p}) \frac{\partial}{\partial \mathbf{g}} t^{\dagger}(\mathbf{g}, \mathbf{g}) \pm \nabla f_{\mu}^{\alpha}(\mathbf{r}, \mathbf{p}) f_{\beta}^{\mu}\left(\mathbf{r}, \mathbf{p}_{1}\right) \frac{\partial}{\partial \mathbf{g}} t^{\dagger}(\mathbf{g},-\mathbf{g}) \\
& +f_{\beta}^{\alpha}(\mathbf{r}, \mathbf{p}) \nabla f_{\mu}^{\mu}\left(\mathbf{r}, \mathbf{p}_{1}\right)\left[t_{\mathbf{q}}^{\dagger}(\mathbf{g}+\mathbf{q}, \mathbf{g}-\mathbf{q})-t_{\mathbf{q}}(\mathbf{g}+\mathbf{q}, \mathbf{g}-\mathbf{q})\right] \\
& \left. \pm f_{\mu}^{\alpha}(\mathbf{r}, \mathbf{p}) \nabla f_{\beta}^{\mu}\left(\mathbf{r}, \mathbf{p}_{1}\right) t_{\mathbf{q}}^{\dagger}(\mathbf{g}+\mathbf{q},-\mathbf{g}+\mathbf{q})-\nabla f_{\mu}^{\alpha}\left(\mathbf{r}, \mathbf{p}_{1}\right) t_{\mathbf{q}}(-\mathbf{g}-\mathbf{q}, \mathbf{g}-\mathbf{q})\right]+\cdots
\end{aligned}
$$

where we have introduced the notation

$$
t_{\mathbf{q}}=\left.\frac{\partial}{\partial \mathbf{q}} t(\mathbf{q})\right|_{\mathbf{q} \rightarrow 0}
$$

Note that there are two completely different types of terms in Eq. (4.6). One of these types corresponds to the zerosound contributions (see below) which were taken into account by the Fermi-liquid-like approach ${ }^{2,4-6}$ and were certainly absent within the local classical approach. ${ }^{7}$ The second type of term (terms $t_{\mathrm{q}}$ ) is rather anomalous: In principle, the two-particle $T$ matrix $t\left(\mathbf{g}^{\prime}, \mathbf{g}\right)$ is well-defined on the mass surface $|\mathbf{g}|=\left|\mathbf{g}^{\prime}\right|$, which corresponds to the energy conservation throughout the collisions; however, the derivatives $\partial / \partial \mathbf{q}$ in Eq. (4.6) include the derivatives over the normal to the mass surface and the corresponding imaginary off-shell contributions.

The terms linear in gradients (4.6) may be easily incorporated into the kinetic equation (3.10) using the expansion of the operators $\hat{f}$ over the system of operators $\hat{l}_{i},(3.5)-(3.7)$. As a result, there appear additional terms in the left-hand side of Eq. (3.10)

$$
\begin{aligned}
& -k_{\alpha} S_{\alpha} \boldsymbol{v}(\mathbf{p})-k_{\alpha} \int d \mathbf{p}_{1} \hat{R}_{\alpha} \boldsymbol{v}\left(\mathbf{p}_{1}\right), \\
& \hat{S}=S_{k i}=\left(c_{j} \delta_{i k} \frac{\partial}{\partial \mathbf{g}} t_{3} \pm a_{i j k} \frac{\partial}{\partial \mathbf{g}} t_{2}\right) n_{j}^{(0)}\left(\mathbf{p}_{1}\right), \\
& \hat{\mathbf{R}} \equiv \mathbf{R}_{k i}=c_{i} \delta_{j k} \frac{\partial}{\partial \mathbf{p}} n_{j}^{(0)} t_{3}+a_{i j k} \frac{\partial}{\partial \mathbf{p}} n_{j}^{(0)} t_{2} \pm n_{j}^{(0)}(\mathbf{p})\left(a_{j i k} t_{\mathbf{q}}^{\dagger}(\mathbf{g}+\mathbf{q},-\mathbf{g}+\mathbf{q})+a_{i j k} t_{\mathbf{q}}(-\mathbf{g}+\mathbf{q}, \mathbf{g}+\mathbf{q}),\right. \\
& 2 t_{3}=t^{\dagger}(\mathbf{g}, \mathbf{g})+t(\mathbf{g}, \mathbf{g}),
\end{aligned}
$$

where we have again used the optical theorem (2.2) and have not presented the terms quadratic in $t$.

The matrix $\hat{A}_{1}$ is obtained by momentum integration of Eq. (4.8) using the variational approach (4.2):

$$
\begin{aligned}
\hat{A}_{1} \equiv A_{1 k i}=\left(9 T N_{i}^{(0)}\right)^{-1}(2 \pi \hbar)^{-3} \int d \mathbf{p} d \mathbf{p}_{1} & {\left[\frac{p p_{1}}{M T}\left(c_{i} \delta_{j k}-c_{j} \delta_{i k}\right) t_{3}^{(1)} \pm a_{j i k}\left(3 p_{1}^{2} \theta_{0}^{\dagger}-p p_{1} \theta_{1}^{\dagger}\right)\right.} \\
& \left.+a_{i j k}\left(3 p_{1}^{2} \theta_{0}-p p_{1} \theta_{1}\right)\right] n_{i}^{(0)}\left(\mathbf{p}_{1}\right) n_{j}^{(0)}(\mathbf{p}),
\end{aligned}
$$

where the angular harmonics $t_{3}^{(n)}, \theta_{n}, \theta_{n}^{\dagger}$ for the functions $t_{3}, \pm t_{\mathbf{q}}(-\mathbf{g}+\mathbf{q}, \mathbf{g}+\mathbf{q})=\mathbf{g} \theta\left(\mathbf{p}, \mathbf{p}_{1}\right)$, and $\pm t_{\mathbf{q}}^{\dagger}(\mathbf{g}+\mathbf{q},-\mathbf{g}$ $+\mathbf{q})=\mathbf{g} \theta^{\dagger}$ are introduced in the same way as in Eq. (3.18).

The matrix $\hat{A}_{2}$ is evaluated multiplying Eq. (4.8) by p/M with consequent momentum integration:

$$
\begin{aligned}
\hat{A}_{2}=A_{2 k i}=\left(9 N_{i}^{(0)} T\right)^{-1}(2 \pi \hbar)^{-3} \int & d \mathbf{p} \\
\times & d \mathbf{p}_{1} n_{i}^{(0)}\left(\mathbf{p}_{1}\right) n_{j}^{(0)}(\mathbf{p}) \\
\times & \left\{\frac{p^{2}}{M T}\left[3 c_{i} \delta_{j k} t_{3}^{(0)}-\frac{p_{1}}{p} c_{j} \delta_{i k} t_{3}^{(1)}+a_{i j k}\left[3 t_{2}^{(0)}-\frac{p_{1}}{p} t_{2}^{(1)}\right]\right]\right. \\
& \left.+p p_{1}\left(\theta_{1}^{\dagger} a_{j i k}+\theta_{1} a_{i j k}\right)-3 p^{2}\left(a_{j i k} \theta_{0}^{\dagger}+a_{i j k} \theta_{0}\right)\right\} .
\end{aligned}
$$


The second-order gradient terms in the right-hand side of Eq. (2.12) in accordance with Eqs. (2.13) and (4.5) take the form

$$
\begin{aligned}
-(i \hbar / 2)(2 \pi \hbar)^{3} \int d \mathbf{p}_{1}[ & {\left[-t(\mathbf{g}, \mathbf{g}) \frac{\partial^{2}}{\partial p_{i} \partial p_{k}} f_{\beta}^{\alpha}(\mathbf{r}, \mathbf{p})+2 t_{\mathbf{q}_{i}}(\mathbf{g}+\mathbf{q}, \mathbf{g}-\mathbf{q}) \frac{\partial}{\partial p_{k}} f_{\beta}^{\alpha}(\mathbf{r}, \mathbf{p})\right] \frac{\partial^{2}}{\partial r_{i} \partial r_{k}} f_{\mu}^{\mu}\left(\mathbf{r}, \mathbf{p}_{1}\right) } \\
& \mp \frac{\partial^{2}}{\partial r_{i} \partial r_{k}} f_{\mu}^{\alpha}\left(\mathbf{r}, \mathbf{p}_{1}\right)\left[t(-\mathbf{g}, \mathbf{g}) \frac{\partial^{2}}{\partial p_{i} \partial p_{k}} f_{\beta}^{\mu}(\mathbf{r}, \mathbf{p})-2 t_{\mathbf{q}_{i}}(-\mathbf{g}-\mathbf{q}, \mathbf{g}-\mathbf{q}) \frac{\partial}{\partial p_{k}} f_{\beta}^{\mu}(\mathbf{r}, \mathbf{p})\right] \\
& +\frac{\partial^{2}}{\partial r_{i} \partial r_{k}} f_{\beta}^{\alpha}(\mathbf{r}, \mathbf{p}) f_{\mu}^{\mu}\left(\mathbf{r}, \mathbf{p}_{1}\right) \frac{\partial^{2}}{\partial p_{i} \partial p_{k}} t^{\dagger}(\mathbf{g}, \mathbf{g}) \pm \frac{\partial^{2}}{\partial r_{i} \partial r_{k}} f_{\mu}^{\alpha}(\mathbf{r}, \mathbf{p}) f_{\beta}^{\mu}\left(\mathbf{r}, \mathbf{p}_{1}\right) \frac{\partial^{2}}{\partial g_{i} \partial g_{k}} t^{\dagger}(\mathbf{g},-\mathbf{g}) \\
& +f_{\beta}^{\alpha}(\mathbf{r}, \mathbf{p}) \frac{\partial^{2}}{\partial r_{i} \partial r_{k}} f_{\mu}^{\mu}\left(\mathbf{r}, \mathbf{p}_{1}\right)\left[t_{\mathbf{q}_{i} \mathbf{q}_{k}}^{\dagger}(\mathbf{g}+\mathbf{q}, \mathbf{g}-\mathbf{q})-t_{\mathbf{q}_{i} \mathbf{q}_{k}}(\mathbf{g}+\mathbf{q}, \mathbf{g}-\mathbf{q})\right] \\
& \pm f_{\mu}^{\alpha}(\mathbf{r}, \mathbf{p}) \frac{\partial^{2}}{\partial r_{i} \partial r_{k}} f_{\mu}^{\mu}\left(\mathbf{r}, \mathbf{p}_{1}\right) t_{\mathbf{q}_{i} \mathbf{q}_{k}}^{\dagger}(\mathbf{g}+\mathbf{q},-\mathbf{g}+\mathbf{q}) \\
& \left.\mp \frac{\partial^{2}}{\partial r_{i} \partial r_{k}} f_{\mu}^{\alpha}\left(\mathbf{r}, \mathbf{p}_{1}\right) f_{\beta}^{\mu}(\mathbf{r}, \mathbf{p}) t_{\mathbf{q}_{i} \mathbf{q}_{k}}(-\mathbf{g}+\mathbf{q}, \mathbf{g}+\mathbf{q})\right],
\end{aligned}
$$

where $t_{\mathbf{q}_{i} \mathbf{q}_{k}}=\partial^{2} t(\mathbf{q}) / \partial q_{i} \partial q_{k}(\mathbf{q} \rightarrow 0)$. The terms in Eq. (4.11) give the following contribution linear in $t$ to the left-hand side of Eq. (3.10):

$$
\begin{aligned}
& \hbar k_{\alpha} k_{\beta}\left[\widehat{S}_{\alpha \beta} \boldsymbol{v}(\mathbf{p})+\int d \mathbf{p}_{1} \widehat{R}_{\alpha \beta} \boldsymbol{v}\left(\mathbf{p}_{1}\right)\right], \\
& \hat{S}_{\alpha \beta} \equiv S_{k i, \alpha \beta}=\int d \mathbf{p}_{1} n_{j}^{(0)}\left(\mathbf{p}_{1}\right)\left[c_{j} \delta_{i k} \partial^{2} t_{3} / \partial g_{\alpha} \partial g_{\beta}+a_{i j k} \partial^{2} t_{2} / \partial g_{\alpha} \partial g_{\beta}\right] \text {, } \\
& \widehat{R}_{\alpha \beta} \equiv R_{k i, \alpha \beta}=c_{i} \delta_{j k}\left[-t_{3} \partial^{2} n_{j}^{(0)} / \partial p_{\alpha} \partial p_{\beta}+2 t_{\mathbf{q}_{\alpha}}(\mathbf{g}+\mathbf{q}, \mathbf{g}-\mathbf{q}) \partial n_{j}^{(0)} / \partial p_{\beta}\right] \\
& \mp a_{i j k}\left[ \pm t_{2} \partial^{2} n_{j}^{(0)} / \partial p_{\alpha} \partial p_{\beta}+2 t_{\mathbf{q}_{\alpha}}(-\mathbf{g}+\mathbf{q}, \mathbf{g}+\mathbf{q}) \partial n_{j}^{(0)} / \partial p_{\beta}\right] \\
& \pm a_{j i k} n_{j}^{(0)}(\mathbf{p}) t_{\mathbf{q}_{\alpha} \mathbf{q}_{\beta}}^{\dagger}(\mathbf{g}+\mathbf{q},-\mathbf{g}+\mathbf{q}) \mp a_{i j k} n_{j}^{(0)}(\mathbf{p}) t_{\mathbf{q}_{\alpha} \mathbf{q}_{\beta}}(-\mathbf{g}+\mathbf{q}, \mathbf{g}+\mathbf{q}) .
\end{aligned}
$$

The matrix $\widehat{A}_{3}$ is obtained by the momentum integrations of Eq. (4.12):

$$
\begin{aligned}
& \hat{A}_{3} \equiv A_{3 k i} \\
& =-\left(M / 9 N_{i}^{(0)} T\right)(2 \pi \hbar)^{-3} \\
& \quad \times \int d \mathbf{p} d \mathbf{p}_{1}\left(( 1 / M T ) ( c _ { j } \delta _ { i k } - c _ { i } \delta _ { j k } ) \left[\left(3 p^{2} / M T-9\right) t_{3}^{(0)}-\left(2 c_{i} \delta_{j k} / M T\right)\left(p p_{1} \eta_{1}-3 p^{2} \eta_{0}\right)\right.\right. \\
& \left.+\left(2 a_{i j k} / M T\right)\left(p p_{1} \theta_{1}-3 p^{2} \theta_{0}\right)\right] \\
& +a_{i j k}\left\{\phi_{0}+3\left[\left(p^{2}+p_{1}^{2}\right) / M T\right] \Psi_{0}-\left(2 p p_{1} / M T\right) \Psi_{1}\right\} \\
& \left.\quad \mp a_{i j k}\left\{\phi_{0}^{\dagger}+3\left[\left(p^{2}+p_{1}^{2}\right) / M T\right] \Psi_{0}^{\dagger}-\left(2 p p_{1} / M T\right) \Psi_{1}^{\dagger}\right\}\right) n_{i}^{(0)}\left(\mathbf{p}_{1}\right) n_{j}^{(0)}(\mathbf{p}),
\end{aligned}
$$

with the notations

$$
\begin{aligned}
& \pm t_{\mathbf{q}_{\alpha} \mathbf{q}_{\beta}}(g+q,-\mathbf{g}+\mathbf{q})=\left(g_{\alpha} g_{\beta} / M T\right) \sum_{n} \Psi_{n} P_{n}(\cos \chi)+\delta_{\alpha \beta} \sum_{n} \phi_{n} P_{n}(\cos \chi), \\
& t_{\mathbf{q}}(\mathbf{g}+\mathbf{q}, \mathbf{g}-\mathbf{q})+t_{\mathbf{q}}^{\dagger}(\mathbf{g}-\mathbf{q}, \mathbf{g}+\mathbf{q})=2 \mathbf{g} \sum_{n} \eta_{n} P_{n}(\cos \chi) .
\end{aligned}
$$

Matrices $\hat{A}(4.9),(4.10)$, and (4.13) provide one with nonlocal corrections to the spectrum (4.4) and to the corresponding diffusion equation. For dilute Boltzmann gas these corrections have the order of magnitude

$$
\hbar \bar{\Omega}^{(i)} / T \sim\left(a_{0}^{3} N\right)\left(\Lambda / a_{0}\right)^{2} \sim\left(T_{0} / T\right) a_{0} N^{1 / 3},
$$

where $\Lambda$ is the de Broglie wavelength of particles, $T_{0}$ is the degeneracy temperature, and $a_{0}$ is the scattering length. Certainly in most of the dilute Boltzmann gases such corrections are small. However, these corrections are of the same order of magnitude as the corrections provided by more accurate derivations of the kinetic equation (2.12) and decoupling of correlations (for example, as the density corrections to the internal frequency $\bar{\Omega}^{(i)}$ ). As a result, both types of corrections must be taken into account simultaneously.

Due to the smallness of nonlocal corrections, the spectrum (4.4) may be rewritten as 


$$
\begin{aligned}
& \operatorname{Det} \| \lambda_{s}\left(\omega_{s}^{(0)} \hat{I}-\widehat{\Omega}^{(0)}\right)+k^{2}(T / M)\left[\hat{I}+\left(\omega_{s}^{(0)} \hat{I}-\widehat{\Omega}^{(0)}+\hat{\bar{\Omega}}^{(i)}-i \hat{\tau}^{-1}\right)^{-1}\left(\omega_{s}^{(0)} \hat{I}-\widehat{\Omega}^{(0)}-\lambda_{s} \hat{I}\right)\right. \\
& -\lambda_{s} \hat{A}_{3}+\widehat{A}_{1}\left(\omega_{s}^{(0)} \hat{I}-\hat{\Omega}^{(0)}+\hat{\bar{\Omega}}^{(i)}-i \hat{\tau}^{-1}\right)^{-1}\left(\omega_{s}^{(0)} \hat{I}-\widehat{\Omega}^{(0)}-\lambda_{s} \hat{I}\right) \\
& \left.+\left(\omega_{s}^{(0)} \hat{I}-\widehat{\Omega}^{(0)}+\hat{\bar{\Omega}}^{(i)}-i \hat{\tau}^{-1}\right)\left(-\omega_{s}^{(0)} \hat{A}_{1}+\widehat{A}_{1} \hat{\Omega}^{(0)}-\lambda_{s} \hat{A}_{2}\right)\right] \|=0 .
\end{aligned}
$$

Let us briefly discuss the limits of applicability of the Fermi-liquid-like approach to the phenomena in question. Such an approach was used beginning from the first papers on spin waves in spin-polarized Boltzmann gases ${ }^{4}$ (see also Ref. 2), and it provided one with the correct spectrum of spin waves which coincided with the spectrum of spin waves obtained by the classical kinetic approach analogous to that represented above.

It has been known for a rather long time (see Ref. 3 and references therein), that an approach of the Fermiliquid type (the so-called concept of "statistical quasiparticles") allows one to obtain the virial coefficient of a Boltzmann gas and all corresponding thermodynamic contributions. Moreover, it was shown, ${ }^{6}$ that inclusion in the Hamiltonian of the Fermi-liquid term

$$
\delta H_{\beta}^{\alpha}=\int\left[d \mathbf{p}_{1} \frac{1}{(2 \pi \hbar)^{3}}\right] F_{\beta v}^{\alpha \mu}\left(\mathbf{p}, \mathbf{p}_{1}\right) f_{\mu}^{v}\left(\mathbf{p}_{1}\right),
$$

with the accurately symmetrized Fermi-liquid function $\widehat{F}$, made it possible to obtain the correct spectrum of spin waves in spin-polarized quantum $a_{0} / \Lambda \ll 1$ gases for all temperatures from the Boltzmann region down to the degenerate one, on the basis of the kinetic equation for the Wigner distribution function,

$$
\partial_{t} \hat{f}+(i / \hbar)[\hat{H}+\delta \hat{H}, \hat{f}]=-\hat{\Gamma}(\hat{f})
$$

( $-\hat{\Gamma}$ is the collision operator). Within this approach one needs only to neglect the insignificant gradient (zerosound) term and use adequate relations between $\widehat{F}$ and $\hat{T}$ in order to see that the kinetic equation (4.17) reduces to (2.16).

The real question is to what extent Eqs. (4.16) and (4.17) give the "exact" kinetic equation and the collective mode spectrum with nonlocal corrections (4.6) and (4.11).

The direct application of Eqs. (4.16) and (4.17) provides one $\mathrm{e}^{2,4,6}$ with some nonlocal terms (zero-sound terms) of the type of two first terms in the brackets in Eq. (4.6); these terms are certainly absent in a purely local approach. $^{7}$ The approach on the basis of Eqs. (4.16) and (4.17), contains enough signs of nonlocality to provide a possibility of a fit to thermodynamic data, ${ }^{12}$ but cannot be considered as a consistent nonlocal approach.

In order to obtain accurate gradient corrections within the Fermi-liquid approach one needs to use the nonlocal expressions for the energy

$$
E=F_{\beta v}^{\alpha \mu}\left(\rho_{\alpha}^{\beta} \rho_{\mu}^{v} \pm \rho_{\mu}^{\beta} \rho_{\alpha}^{v}\right),
$$

nonlocal correction to the Hamiltonian,

$$
\delta H_{\beta}^{\alpha}=\delta E / \delta \rho_{\alpha}^{\beta}=\left(F_{\beta v}^{\alpha \mu}+F_{v \beta}^{\mu \alpha} \pm F_{v \beta}^{\alpha \mu} \pm F_{\beta v}^{\mu \alpha}\right) \rho_{\mu}^{v},
$$

and the corresponding nonlocal corrections to the kinetic equation (here indices correspond not only to the internal indices, but also to the space or momenta states). Such a kinetic equation with nonlocal Fermi-liquid interaction has the same form as Eq. (2.12), and makes it possible to relate the nonlocal Fermi-liquid interaction for gases with the elements of $T$ matrix and their derivatives. In the same way it is desirable to determine relations between the nonlocal Fermi-liquid interaction in dense Fermiliquids [which may be introduced with the help of additional phenomenological $f$ function (Ref. 13)] with an exact vertex.

\section{SUMMARY}

The main problem studied above is a propagation of collective modes in Boltzmann gases of particles with arbitrary internal degrees of freedom. This problem has two quite different aspects. The first concerns the relative strength of coherent and noncoherent interactions. In other words, it is a question of damping of collective modes. This question was not considered above, but still it is necessary to make some concluding remarks. Unfortunately, in most of the cases the damping (imaginary part of the frequency) is of the same order as, or even more important than, the molecular field (real part of the frequency) which prevents the observation of collective effects. From this point of view the possibility of observation of collective oscillation is restricted either to some specific cases of strong molecular field and/or low damping (such as the case of plasma waves, when one deals with strong long-range Coulomb interaction and small scattering cross sections) or to a general case of "quantum gases" $\Lambda / a_{0} \gtrsim 1$. The last regime corresponds to the low-temperature gases. For most particles that means also the extremely low density of a gas, and the consequent difficulties for experiment.

There is also one additional dissipation mechanism very important for Boltzmann gases-Landau damping. Usually in Boltzmann gases Landau damping prevents the propagation of collective nonhydrodynamic modes with linear spectrum, but in the above case of collective modes with quadratic spectrum $\omega \propto k^{2}$ the Landau damping becomes important only beyond the $k^{2}$ region (see Ref. 2).

The second aspect of the problem in question concerns the mere existence of collective effects. This question was studied above in detail, and now it is possible to formulate the general conditions for the appearance of collective effects in gases.

(1) The coherent oscillations are caused by the existence of molecular field and the corresponding internal frequency $\bar{\Omega}^{(i)}$. At the same time, the nonzero internal frequency $\bar{\Omega}^{(i)}$ provides the $k^{2}$ type of spectrum thus making the Landau damping insignificant.

(2) In the gases with the "exchange" interaction (interaction independent of internal states of colliding particles) the appearance of a nonzero internal frequency $\bar{\Omega}^{(i)}$ 
is provided only by a nonzero value of the linearized commutator $\left[\hat{f}(\mathbf{p}), \hat{f}\left(\mathbf{p}_{1}\right)\right]$ of single-particle distributions which are matrices in internal states. Since the commutator of diagonal matrices is equal to zero, and the equilibrium density matrix is diagonal in internal states, this commutator has a nonzero value only for off-diagonal (transverse) perturbations of distributions.

(3) Moreover, this commutator being linearized in small perturbations is nonzero only if the equilibrium distribution is not reduced to the unitary matrix in internal states. This means that equilibrium populations of internal states must be different from each other, or, in other words, the equilibrium state must be "polarized" in the sense of internal states. This "polarization" may be provided either by temperature factor (in the case of nondegenerate internal levels; such a "polarization" is a truly equilibrium one), or by some external source, e.g., by some sort of pumping. In the latter case such an artificial nonequilibrium "polarization" of unperturbed state is conserved if the interaction is an exchange one, and the collective effects may be observed. The "internal" frequency $\bar{\Omega}^{(i)}$ is proportional to the "polarization."

(4) The simplest situation corresponds to equidistant internal energy levels. Then, if the populations of successive levels differ by the same constant there always exists a branch of the collective mode spectrum exactly the same as for spin waves in spin-polarized gases. For equilibrium polarization that is temperature ensured this condition is met if the separation between equidistant levels is much smaller than the temperature as in (3.25). Certainly all the types of gases of two-level particles always correspond to this case.

(5) The damping of coherent collective modes in Boltzmann gases of particles with internal structure is due mainly to the usual collision relaxation responsible for diffusion (the difference between "longitudinal" and "transverse" diffusion relaxation times ${ }^{6}$ is not very important for Boltzmann gases ${ }^{14}$ ). The damping may be small at low temperatures and/or small scattering amplitudes.

(6) The internal energy levels must be very well resolved and possess very small imaginary parts (natural widths) in order to make the observation of rather weak collective effects possible.

Apart from the above conditions, in this paper we have also expressed the collective-mode spectrum (its real part) via the elements of the scattering $T$ matrix. The very important point is that more accurate and sophisticated schemes of derivation of the kinetic equation, e.g., involving the density corrections to internal frequency $\bar{\Omega}^{(i)}$ (such a scheme has been recently developed in Ref. 15), must be accompanied by simultaneous evaluation of nonlocal and gradient terms thus involving zero-sound and off-shell contributions. The results clearly demonstrate how nonlocal terms become more and more important with increasing density. These results also demonstrate the shortcomings of both classical kinetic and usual Fermi-liquid approaches to transverse oscillations.

The above results provide one with the symmetry conditions for the observation of collective effects in classical Boltzmann gases of particles with arbitrary internal degrees of freedom. Still there is an additional question of how to observe these "transverse" oscillations in different particular cases. In some cases the experimental methods seem to be obvious - for example, in the cases of lowtemperature gases of molecules with rotational levels (e.g., low-temperature ortho- $\mathrm{H}_{2}$ ) the observations may be done by the usual magnetic methods. In other cases the experimental techniques of excitation and detection of collective oscillations corresponding to the collectivized transitions between internal energy levels are not so obvious (for example, for an ortho-para conversion wave in hydrogen gas, or for a wave corresponding to transitions between bending states of linear molecules).

\section{ACKNOWLEDGMENTS}

I would like to thank Professor F. Laloë (Paris) for numerous discussions and correspondence on different aspects of kinetic equations for Boltzmann gases. I am grateful to Professor S. Stringari for hospitality at the University of Trento.
${ }^{1}$ H. Moraal, Phys. Rep. 17, 225 (1975).

${ }^{2}$ A. E. Meyerovich, Progress in Low Temperature Physics, edited by D. F. Brewer (North-Holland, Amsterdam, 1987), Vol. 11, pp. 1-73; A. E. Meyerovich, Anomalous Phases of ${ }^{3} \mathrm{He}$, edited by W. P. Halperin and L. P. Pitaevski (North-Holland, Amsterdam, in press).

${ }^{3}$ R. Balian and C. de Domnicis, Ann. Phys. (N.Y.) 62, 229 (1971); J. M. Luttinger and Y. T. Liu, ibid. 80, 1 (1973); M. B. Vetrovec, and G. M. Carneiro, Phys. Rev. B 22, 1250 (1980).

${ }^{4}$ E. P. Bashkin and A. E. Meyerovich, Zh. Eksp. Toer. Fiz. 77, 383 (1979) [Sov. Phys._JETP 50, 196 (1979)]; Adv. Phys. 30, 1 (1981).

${ }^{5}$ E. P. Bashkin, Pis'ma Zh. Eksp. Teor. Fiz. 33, 11 (1981) [JETP Lett. 33, 8 (1981)].

${ }^{6}$ A. E. Meyerovich, Phys. Lett. A107, 177 (1985).

${ }^{7}$ C. Lhuillier and F. Laloë, J. Phys. (Paris) 43, 197 (1982); 43,
225 (1982); 44, 1 (1983).

${ }^{8}$ S. Hess, Z. Naturforsch. 22, 1871 (1967).

${ }^{9}$ J.-P. Bouchaud and C. J. Lhuillier, J. Phys. (Paris) 11, 1781 (1985).

${ }^{10}$ F. M. Chen and R. F. Snider, J. Chem. Phys. 46, 3937 (1967); 48, 3185 (1968); 50, 4082 (1969).

${ }^{11}$ A. J. Leggett, J. Phys. C 3, 448 (1970).

${ }^{12}$ E. P. Bashkin, Zh. Eksp. Teor. Fiz. 93, 1856 (1987). [Sov. Phys. - JETP 66, 482 (1987)].

${ }^{13}$ A. E. Meyerovich, J. Low Temp. Phys. 53, 487 (1983).

${ }^{14}$ It was demonstrated by F. Laloë; private communications. Later it was confirmed in Ref. 15.

${ }^{15}$ G. Tastevin, P. J. Nacher, and F. Laloë, Proceedings of the $3 r d$ International Conference on Spin-Polarized Quantum Systems (Torino, Italy, 1988), edited by S. Stringari (World Scientific, Sinagpore, in press); F. Laloë (private communication). 